\title{
DISCOVERY: a study examining the prevalence of transthyretin mutations in subjects suspected of having cardiac amyloidosis
}

\author{
Olakunle Akinboboye ${ }^{1 *}$, Karthik Ananthasubramaniam², Amir Malik ${ }^{3}$, Alberta Warner ${ }^{4}$, Verena Karsten ${ }^{5}$, \\ Thibaud Damy ${ }^{6}$, Herman A Taylor ${ }^{7}$, Mathew S Maurer ${ }^{8}$ \\ From First European Congress on Hereditary ATTR amyloidosis \\ Paris, France. 2-3 November 2015
}

\section{Background}

Cardiac amyloidosis (CA) is caused by extracellular myocardial deposition of either immunoglobulin light-chain (AL) or transthyretin (ATTR) fibrils. Two forms of ATTR CA cause life-threatening cardiomyopathy: an inherited form arising from misfolding of mutated ATTR (familial amyloid cardiomyopathy [FAC]) and a sporadic form caused by wild-type ATTR (senile systemic amyloidosis [SSA]). More than half of over 100 reported ATTR mutations are associated with FAC. The most common mutation in the US is Val122Ile found in 3-4\% of African Americans (AA). FAC can be difficult to recognize clinically and is likely under diagnosed. The DISCOVERY study aims to determine the prevalence of TTR mutations and FAC diagnosis in a cohort of patients (pts) with clinical features suggestive of CA.

\section{Methods}

This is a prospective, multi-center study in adults with two or more of the following eligibility criteria: heart failure signs and symptoms, intraventricular septal thickness (IVS) of $>12 \mathrm{~mm}$, LV diastolic dysfunction, low voltage ECG, or history of carpal tunnel disease (CTD). DNA from blood samples is used for sequencing of the TTR gene coding regions by a central lab. Assessments in pts with TTR mutations include cardiac biomarkers, echocardiogram and optional abdominal fat pat aspiration and 6-minute walk test. Descriptive statistics will be utilized.

\section{Results}

As of May 2015, 146 pts have been enrolled. At baseline, the mean (Std) age of pts is 64 (13) yrs, $61 \%$ are

${ }^{1}$ Queens Heart Institute, Medicine, 11422, Rosedale, USA

Full list of author information is available at the end of the article men and $66 \%$ are AA. A total of $14(10 \%)$ pts had a Val122Ile mutation and $1 \mathrm{pt}$ had a novel mutation Arg103His. The Gly6Ser polymorphism was found in 8 (5\%) pts. The Val122Ile cohort consisted of $40 \%$ males with a mean (StD) age of 66 (16). Heart failure signs and symptoms and IVS > $12 \mathrm{~mm}$ was reported in $71 \%$ and $79 \%$ of Val122IIle pts respectively. The majority of pts had NYHA class II (56\%) and III (22\%) heart failure, $21 \%$ had low voltage ECGs and 14\% had CTD.

\section{Conclusions}

These preliminary data suggest that approximately $10 \%$ of pts with clinical and/or radiologic findings suggestive of cardiac amyloidosis have a pathogenic TTR mutation which could potentially lead to a diagnosis of FAC. Additional data on clinical features and tissue diagnosis of FAC in these pts will be presented.

\begin{abstract}
Authors' details
${ }^{1}$ Queens Heart Institute, Medicine, 11422, Rosedale, USA. ${ }^{2}$ Henry Ford Hospital, Heart and Vascular Institute, 48202, Detroit, USA. ${ }^{3}$ Heart Center of North Texas, Cardiology, 76104, Fort Worth, USA. ${ }^{4}$ Los Angeles Veterans Affairs Health Care System, Echo lab and HF service, 90073, Los Angeles, USA. ${ }^{5}$ Alnylam Pharmaceuticals, Clinical Development, 02142, Cambridge, USA. ${ }^{6}$ APHP, Cardiology and Amyloidosis Mondor Network, 94000, Creteil, France. ${ }^{7}$ Morehouse School of Medicine, Cardiovascular Research Institute, 30310, Atlanta, USA. ${ }^{8}$ Columbia University Medical Center, Medicine, 10032, New Yok, USA.
\end{abstract}

Published: 2 November 2015

\section{doi:10.1186/1750-1172-10-S1-O8}

Cite this article as: Akinboboye et al:: DISCOVERY: a study examining the prevalence of transthyretin mutations in subjects suspected of having cardiac amyloidosis. Orphanet Journal of Rare Diseases 2015 10(Suppl 1):08 